\title{
Extraction of prior grain boundaries from interfaces of martensite based on particular statistics for inter-variant disorientations
}

\author{
A. A. Zisman ${ }^{1,2}$, D. R. Kolomoets ${ }^{1, \dagger}$, N. Yu. Zolotorevsky ${ }^{2}$, S. N. Petrov ${ }^{1,2}$ \\ †Denis_Kolomoets@crism.ru \\ 'NRC "Kurchatov Institute" - CRISM “Prometey", 49 Shpalernaya str., St.-Petersburg, 191015, Russia \\ ${ }^{2}$ Peter the Great St.-Petersburg Polytechnic University, 29 Polytekhnicheskaya str., St.-Petersburg, 195251, Russia
}

\begin{abstract}
The paper considers grounds and limitations of various ways to reveal prior austenite grain boundaries (PAGB) in martensite in terms of EBSD data and a predetermined orientation relationship (OR). In order to improve this reconstruction, a novel approach is proposed that extracts PAGB from a network of interfacial disorientations rather than orientations of transformed crystals. Thus, errors due to non-uniformity of deformed austenite grains are excluded while essentially diminishing computational expenses. Special attention is paid to special $\left(\sum=3\right)$ twin PAGB because a notable part of then would be lost when conventionally discarding all combinations $\left(V_{1} / V_{i}, i=2,3, \ldots, 24\right)$ of OR variants. Instead a limited set $(i=2-6$ and 8$)$ of variant couples most frequent in martensite is considered. Although such a procedure occasionally admits wrong segments of PAGB and still partly discards their twin parts, a simple correction of such errors is possible owing to very low probability to meet them at successive segments of prior boundaries. The algorithm is verified on low carbon martensitic steel where actual PAGB have been previously imaged by the thermal etching in vacuum. In order to quantify their correspondence to PAGB reconstructed by means of EBSD, related length distributions in random intersection of prior grains by straight lines are analyzed.
\end{abstract}

Keywords: prior grain boundary, martensite, orientation relationship, variant pairing.

\section{Introduction}

Reconstruction of prior austenite grain boundaries (PAGB) in martensite or bainite, particularly at the absence of retained austenite, is still a challenging problem. At present, in order to replace procedures of chemical etching or thermal etching in vacuum $[1-3]$ which are not always efficient, this problem is treated in terms of EBSD orientation data. The approach makes use of a fixed (presumed or experimentally determined) orientation relationship (OR) that suggests dissimilar selections of allowed crystal orientations within differently oriented parent grains. Based on this general ground, numerous methods have been proposed to reveal PAGB [4-9]. The paper volume does not allow the authors to consider a reach variety of such efforts revisited elsewhere [10]; however, general troubles of them should be mentioned. In particular, ferrite, when present in the transformed structure, extends over PAGB and thus impedes their detection. Besides, in-grain substructures peculiar to deformed austenite disturb the unique orientation set for transformed crystals in each parent grain. Martensitic steels considered in the present paper have insignificant fractions of ferrite; as to deformed austenite grains, the related nonuniformity weakens at the finer scale of martensite laths or their blocks and thus can be neglected when considering local disorientations rather than a whole array of orientations. For the same reason, for instance, the determination of OR with no reconstruction of prior grains and regardless of their deformation substructure [11] becomes possible. Besides, an angular range of 20 to 50 degrees generally does not contain inter-variant disorientations due to $\mathrm{OR}$ and hence enables one to find about a half of PAGB [12]; to complete their network semi-manually, the image quality (IQ) function is then employed. Apart from the abovementioned issues, the crucial problem, as noted elsewhere $[5-7,9]$, is insufficiency of OR alone to ensure reliable fully automated reconstruction of prior grains because of frequent twin boundaries in austenite. The disorientation across such a special ( 23$)$ PAGB admits adjacent crystals of martensite which comply with OR in both prior grains; in particular, the pairing of OR variants across a prior twin boundary includes near-zero disorientations $[5,6]$ so that the corresponding segments of PAGB are hardly detectable by EBSD. Regardless of combinatorial reasons, the ambiguous boundaries are also attributed to a tendency to diminish the interfacial energy $[5,13]$.

In order to get over the above-mentioned difficulties while avoiding excessive consumption of computational power, three expedients are often applied [6-9] as follows. First, large raw data are properly segmented 
into a set of quasi-uniform units, each involving many original data points. Second, the "two tolerances" strategy is implemented that finds disconnected embryos of the sought structure with certain limitations and then softens the latter to extend this structure. Third, the remaining ambiguity is eliminated by allowance for auxiliary data such as dimensions and shapes of reconstructed grains or IQ of martensite crystals. Our algorithm employs in a sense the same means; however its principal distinction is that both the original structure and the result extracted from the latter consist of linear segments rather than twodimensional domains. Thus, disorientations at potential PAGB instead of all in-grain orientations are analyzed that excludes errors due to non-uniformity of parent grains and, moreover, essentially reduces computational expenses. Some specific aspects of this novel approach are worth noting in advance. One of them is that not only near-zero disorientations of martensite crystals separated by PAGB $[5,6]$ but also a lot of finite inter-variant disorientations complicate the reconstruction procedure in terms of $\mathrm{OR}$. In order to diminish related troubles, we employ a limited selection of variant pairing most probable in martensitic and bainitic steels [14-16]. Besides, to correct occasional errors due to improper increments of PAGB or loss of their special parts, a statistical criterion is applied allowing for negligible probability to meet rare ambiguous segments in succession. The proposed algorithm is verified on low carbon martensitic steel by comparison of reconstructed PAGB to those revealed by the thermal etching in vacuum before the steel quenching; specifically, related length distributions for random intersections of prior grains are considered.

\section{Material and experimental methods}

The proposed algorithm is tested on a hot rolled low carbon martensitic steel (wt.\%: $0.09 \mathrm{C}, 0.35 \mathrm{Mn}, 0.33 \mathrm{Si}$, $5.20(\mathrm{Ni}+\mathrm{Cu}), 1.10(\mathrm{Cr}+\mathrm{Mo}))$ in the as received state. Its $\varnothing 5 \times 10 \mathrm{~mm}$ specimen with a narrow polished section parallel to the cylinder axis has been quenched at the cooling rate of $40{ }^{\circ} \mathrm{C} / \mathrm{s}$ after exposure at $1050{ }^{\circ} \mathrm{C}$ for $200 \mathrm{~s}$ in the vacuum chamber of DIL 805 dilatometer. Groves of about $0.1 \mu \mathrm{m}$ depth formed at PAGB by the selective thermal etching [1-3] provide distinct appearance of equiaxed prior grains on the background of martensite structure that becomes visible due to a specific relief formed by local shears in the $\gamma-\alpha$ transformation. In order to verify depth resolution in the grove imaging on the local scale, SEM Quanta 200 3D has been applied first in a regular SEM mode; then a panoramic image of etched PAGB over an area of $325 \times 330 \mu \mathrm{m}^{2}$ has been obtained by means of optical microscope (OM) ZEISS AXIO Observer-A1m. In order to avoid diffraction errors due to the transformation relief and the boundary grooves, the section is carefully re-polished using colloidal alumina at the finish stage, and EBSD is applied to the same area with the grid step of $0.4 \mu \mathrm{m}$ and EDAX TSL software to derive crystal orientations from diffraction patterns. The considered algorithm reconstructs PAGB using MTEX software and, since the repeated polishing disables local correspondence, appropriate statistical terms are employed to compare the reconstruction result to the thermally etched boundaries.

\section{Prior grain boundaries: Fundamentals and procedure of reconstruction}

\subsection{Orientation relationship and statistics of variant pairing}

Actual OR in martensite vary between those by Kurdjumov-Sachs (KS: $\left.\{111\}_{\gamma} \|\{110\}_{\alpha}, \quad<110\right\rangle_{\gamma} \|<111>_{\alpha}$ ) [17] and Nishiyama-Wasserman (NW: $\{111\}_{\gamma} \|\{110\}_{\alpha}$, $\left.<211\rangle_{\gamma} \|<110\right\rangle_{\alpha}$ ) [18] so that an intermediate OR by Greninger-Troiano (GT) [19] is often closer to reality. To increase accuracy of PAGB reconstruction, we employ the OR specifically determined in the investigated steel according to [11]. This corresponds to Euler's angles $29.7^{\circ}$, $8.8^{\circ}$ and $16.5^{\circ}$ for the relative rotation of elementary cubic cells in the $\gamma-\alpha$ transformation and suggests deviations of $1.5^{\circ}$ and $2.8^{\circ}$ for close packed planes $\{111\}_{\gamma} /\{110\}_{\alpha}$ and directions $\left.<110\rangle_{\gamma} /<111\right\rangle_{\alpha}$, respectively. Owing to some deviation from KS, our OR suggests no similarly of variants separated by twin PAGB; instead, the least disorientation angle at the latter approaches $2.9^{\circ}$.

Regardless of OR observed in martensitic and bainitic steels as well as various statistics of variant pairing $V_{1} / V_{i}$ $(i=2,3, \ldots, 24)$ conventionally listed elsewhere [14-16], there is a persistent set of most probable inter-variant disorientations. Specifically, about $85 \%$ of them in the transformed structure generally correspond to $i=2-6$ and 8 . A fortunate property of this limited set, as shown in what follows, is that it involves only a minor part of OR variant couples possible at twin PAGB. Accordingly, the majority of the latter are saved when one excludes interfaces of such $V_{1} / V_{i}$ in the reconstruction procedure. Although this approach still discards some prior twin boundaries while admitting erroneous PAGB due to about $15 \%$ of ignored variant pairing, related errors can be corrected as described in the next subsection.

\subsection{Ambiguous disorientations in reconstruction of $P A G B$}

Use of the limited selection of variant couples suggests some errors which should be eventually corrected. With actual variations of OR and accuracy of EBSD orientations, the correction means may be considered with any OR, in particular with the classical KS.

Let us consider variants of $\mathrm{KS}(i=1,2, \ldots, 24)$ on one side of a prior $\sum 3$ boundary and respectively rotated variants $(j=1,2, \ldots, 24)$ on its other side. In order to avoid an enormous listing of $24 \times 4=576$ disorientation angles, Table 1 represent a part of them. Rotation axes are omitted here for brevity sake whereas any repeated appearance of the same angle with another axis is indicated by the prime symbol. All represented angles prove to be inter-variant as if adjacent crystals were transformed from austenite within the same parent grain. When extended to $i=24$, each of $1^{\text {st }}$ to $6^{\text {th }}$ columns would involve all possible couples of OR variants; however, zero angles (coinciding variants) appear only in the shown six lines. The same lines extended to $j=24$ would also confine inter-variant angles at $j>7$; differently arranged arrays of such angles repeatedly appear in columns $6+k$, 
$12+k$ and $18+k$ at $k=1,2, \ldots, 6$. The remaining combinations of crystals $(i>6$ at $j>6)$ separated by a prior twin boundary have no inter-variant disorientations.

To simplify analysis, we will assume for the first approximation equivalence of all OR variants in each of prior grains separated by a $\Sigma 3$ boundary. In this case the aboveconsidered data suggest probability of $6 / 24=0.25$ that such grains contain some similarly oriented martensite crystals as previously mentioned in [5]. If they meet, EBSD will become unable to detect a PAGB between them. However, since at each of $j=1,2, \ldots, 6$ only one zero disorientation appears, this kind of error has rather low probability of $(6 / 24) \times(1 / 24) \approx 0.01$ and hence will not seriously affect the reconstruction procedure. On the other hand, according to the same data, there remains significant probability of $(6 / 24) \times 23 / 24+(18 / 24) \times 6 / 24 \approx 0.43$ to meet at twin PAGB a finite inter-variant disorientation. In this case each of adjacent crystals can be formally ascribed to any of two prior grains; to avoid this ambiguity, additional information and special assumptions should be used [7,9] which complicate the automated treatment of EBSD data. As considered in what follows, our algorithm essentially diminishes such complications because the limited set of frequent variant couples $\left(V_{1} / V_{i}, i=2-6\right.$ and 8$)$ excludes only a minor fraction of twin PAGB.

Vectors $\theta_{1 / 2}, \theta_{1 / 3,5}, \theta_{1 / 4}, \theta_{1 / 6}$ and $\theta_{1 / 8}$ of inter-variant disorientations $[8,20]$ most frequent in martensite and bainite are listed in Table 2 where $\theta_{1 / 3,5}$ combines two couples since $\theta_{1 / 5}=-\theta_{1 / 3}$. Except for $\theta_{1 / 8}$, each of related angles appears six times in columns 1 to 6 of Table 1 . If the latter was extended to 24 lines and 24 columns, $\theta_{1 / 8}$ would appear twelve times; specifically, its angle would be found two times within each next six columns at $j>6$ and $i=1,2, \ldots, 6$, as well as within each next six lines at $i>6$ and $j=1,2, \ldots, 6$. Therefore, the probability to meet some pairing of OR variants at a prior twin boundary and hence to exclude the latter from revealed PAGB is reduced to $p_{t w}=(4 \times 6+6 \times 2) / 576 \approx 0.06$. On the other hand, since about $15 \%$ of inter-variant disorientations are not checked, some interfaces of martensite can be erroneously taken for PAGB. Probability $p_{m} \approx 0.15$ of such a mistake notably exceeds $p_{t w}$ and is hardly acceptable. As considered in the next subsection, a simple way to correct such errors is to make use of rather lower probability $p_{m}^{2} \approx 0.02$ of ambiguous variant couples successively situated along an arbitrary PAGB. Similarly, even lesser $p_{t w}^{2} \approx 0.004$ corresponds to inter-variant disorientations at successive segments of a prior twin boundary.

\subsection{Extraction of PAGB from a network of martensite interfaces}

To make use of the previously mentioned experimental OR, interfaces between martensite domains (Fig. 1a) are revealed with a tolerance angle $\theta_{t}^{(0)}$ of two degrees. On the one hand, such $\theta_{t}^{(0)}$ is high enough to comply with realistic accuracy of EBSD; on the other hand, it is sufficiently small to provide a properly refined interfacial network for the PAGB reconstruction.

To exclude inter-variant disorientations, two tolerance angles $\theta_{t}^{(1)}>\theta_{t}^{(0)}$ and $\theta_{t}^{(0)}<\theta_{t}^{(2)}<\theta_{t}^{(1)}$ are employed. As considered below, the higher value $\theta_{t}^{(1)}$ saves a lesser fraction of PAGB whereas $\theta_{t}^{(2)}$ results in their extension. Accordingly, when predetermined on a trial martensite area, $\theta_{t}^{(1)}$ should provide realistic shapes and dimensions of prior grains displayed by about a half of their boundaries; $\theta_{t}^{(2)}$, in turn,

Table 1. Inter-variant disorientation angles $\left[^{\circ}\right]$ of KS orientation relationship at a twin $\left(\sum 3\right) \operatorname{PAGB}\left(V_{i} \rightarrow V_{j}^{(t w)} ; i, j=1,2, \ldots, 24\right)^{1}$.

\begin{tabular}{|c|c|c|c|c|c|c|c|c|c|c|c|c|}
\hline$i$ & 1 & 2 & 3 & 4 & 5 & 6 & 7 & 8 & 9 & 10 & 11 & 12 \\
\hline 1 & 60.0 & 10.5 & 60.0 & 49.5 & 0 & $60.0^{\prime}$ & 14.9 & $50.5^{\prime}$ & $57.2^{\prime}$ & $20.6^{\prime}$ & 51.7 & 47.1 \\
\hline 2 & 49.5 & 60.0 & 10.5 & 60.0 & $60.0^{\prime}$ & 0 & 57.2 & 50.5 & 21.1 & $57.2^{\prime}$ & 47.1 & 20.6 \\
\hline 3 & 0 & $60.0^{\prime}$ & 60.0 & 10.5 & 60.0 & 49.5 & $49.5^{\prime}$ & $10.5^{\prime}$ & 50.5 & $50.5^{\prime}$ & 14.9 & 57.2 \\
\hline 4 & $60.0^{\prime}$ & 0 & 49.5 & 60.0 & 10.5 & 60.0 & $10.5^{\prime}$ & $49.5^{\prime}$ & 57.2 & 14.9 & $50.5^{\prime}$ & 50.5 \\
\hline 5 & 60.0 & 49.5 & 0 & $60.0^{\prime}$ & 60.0 & 10.5 & 50.5 & 57.2 & 20.6 & 47.1 & $57.2^{\prime}$ & 21.1 \\
\hline 6 & 10.5 & 60.0 & $60.0^{\prime}$ & 0 & 49.5 & 60.0 & $50.5^{\prime}$ & 14.9 & 47.1 & 51.7 & $20.6^{\prime}$ & $57.2^{\prime}$ \\
\hline
\end{tabular}

Table 2. Appearance of most probable inter-variant disorientations at twin ( 23 ) PAGB.

\begin{tabular}{|c|ccc|c|}
\hline \multirow{2}{*}{ Couple of KS OR variants } & \multicolumn{2}{|c|}{ Disorientation (angle, $/$ axis) } & \multicolumn{2}{|c|}{ Respective cells $(i / j)$ of Table 1 } \\
\hline$V_{1} / V_{2}$ & $60.0 / 0.58$ & -0.58 & 0.58 & $1 / 6,2 / 5,3 / 2,4 / 1,5 / 4,6 / 3$ \\
\hline$V_{1} / V_{3,5}$ & $60.0 / 0$ & -0.71 & -0.71 & $1 / 1,2 / 2,3 / 3,4 / 4,5 / 5,6 / 6$ \\
\hline$V_{1} / V_{4}$ & $10.5 / 0$ & 0.71 & 0.71 & $1 / 2,2 / 3,3 / 4,4 / 5,5 / 6,6 / 1$ \\
\hline$V_{1} / V_{6}$ & $49.5 / 0$ & 0.71 & 0.71 & $1 / 4,2 / 1,3 / 6,4 / 3,5 / 2,6 / 5$ \\
\hline$V_{1} / V_{8}^{2}$ & $10.5 / 0.58$ & -0.58 & 0.58 & $1 / 20,2 / 19,3 / 8,4 / 7,5 / 14,6 / 13$, \\
\hline & & & $7 / 6,8 / 5,13 / 2,14 / 1,19 / 4,20 / 3$ \\
\hline
\end{tabular}

$1 \quad i>6$ and $j>12$ are omitted for brevity sake; the prime symbol indicates angles repeated with different rotation axes.

2 All $V_{1} / V_{8}$ couples would appear in Table 1 if it was extended to 24 lines and 24 columns. 
is selected to ensure further propagation of the latter while avoiding their avalanching multiplication.

An embryonic fraction of PAGB (Fig. 1b) is extracted first from the original network by excluding all couples $\left(V_{1} / V_{i}\right.$, $i=2,3, \ldots, 24)$ of OR variants with $\theta_{t}^{(1)}$. Any gap of the revealed boundaries over a single interfacial segment is then closed; if there are various ways of closure, the shortest segment is selected. Next, in order to extend the embryonic boundaries (Fig. 1c), $\theta_{t}^{(2)}<\theta_{t}^{(1)}$ is applied. When this expedient no longer adds new segments to PAGB, the same $\theta_{t}^{(2)}$ is used with the limited set $(i=2,3, \ldots, 6,8)$ of variant couples and thus modified PAGB are treated again with the whole set. As in the initial step using $\theta_{t}^{(1)}$, single gaps at the revealed boundaries are closed after each application of $\theta_{t}^{(2)}$. Such iterations are interrupted if the remaining open-ended PAGB undergo virtual bifurcations or stop to extend. Thereafter, special verification procedures for the first two and the next four virtual segments of PAGB are applied at each problematic end of the latter. According to subsection 3.2, these procedures described in what follows should save the majority of prior twin boundaries while discarding the most part of erroneous segments of non-special PAGB.

If no couples of OR variants are found at the first step as well as at the following single segments, the triple junction of PAGB is accepted (Fig. 2a). Otherwise, if the two steps suggest repeated bifurcations at one or both branches (Figs. 2b, 2c or $2 \mathrm{~d}$, respectively), the related segments are dismissed in order to prevent unrealistic multiplication of PAGB.

Next, ambiguous PAGB ends with no first step bifurcations are analyzed according to Fig. 3 where solid and dashed lines correspond to different types of interfacial segments as in Fig. 2. Specifically, a previously lost segment is restored if it has one appropriate continuation at the second step. After each implementation of double step corrections

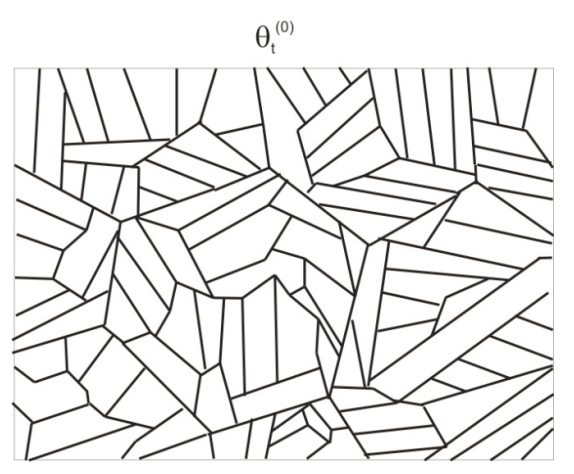

a $\theta^{(1)}>\theta_{t}^{(0)}, V_{1} / V_{i}(i=2,3, \ldots, 24)$

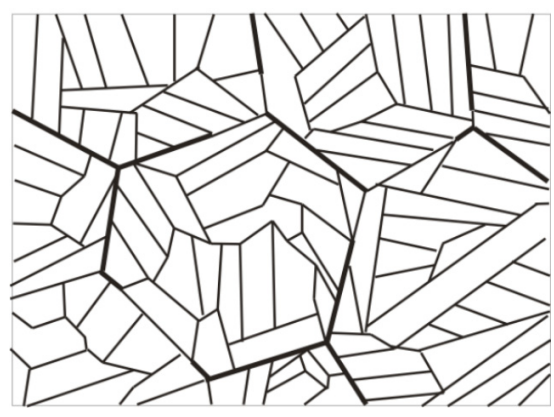

b $\theta_{\mathrm{t}}^{(2)}<\theta_{\mathrm{t}}^{(1)}, \mathrm{V}_{1} N_{\mathrm{i}}(\mathrm{i}=2,3, . ., 24 \& 2,3, \ldots, 6,8)$

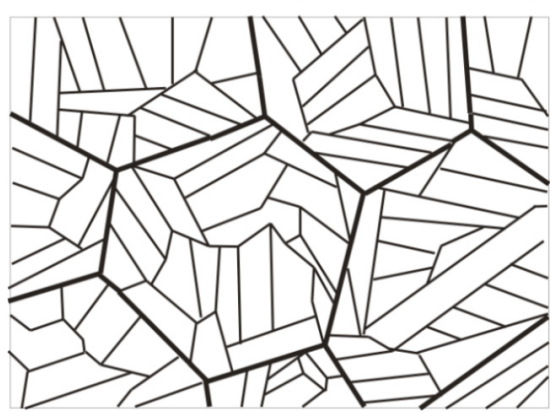

c

Fig. 1. Scheme of PAGB extraction from martensite interfaces: a network of the latter (thin lines) mapped by EBSD (a), disconnected PAGB embryos (thick lines) found by excluding all inter-variant disorientations (b), and a final PAGB totality formed by propagation of the embryos through interfacial bifurcations (c). Tolerance angles and OR variant combinations added over these pictures are used in respective steps of the reconstruction procedure.

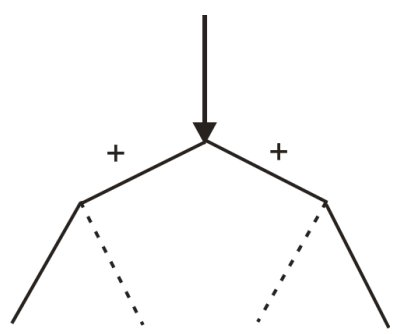

a

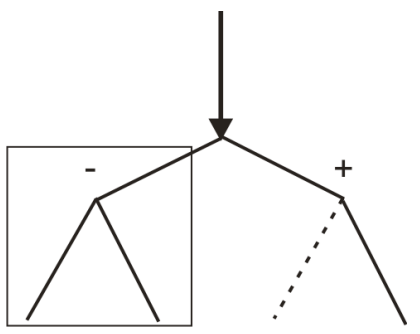

$\mathrm{b}$

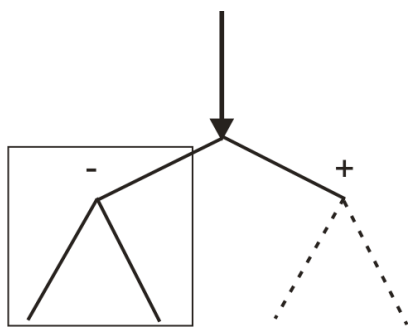

c

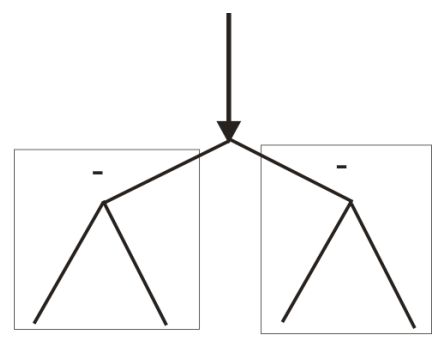

d

Fig. 2. Verification procedure for branching of PAGB (arrows) at junctions of martensite interfaces with arbitrary (solid lines) or inter-variant (dashed lines) disorientations: an acceptable case (a), prohibited cases of branching repeated at one or two next junctions, respectively (b, c and d).

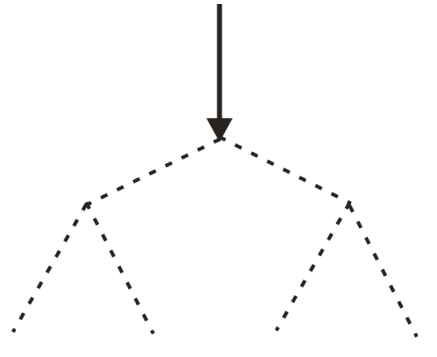

a

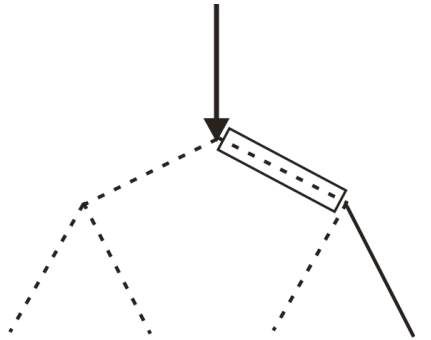

b

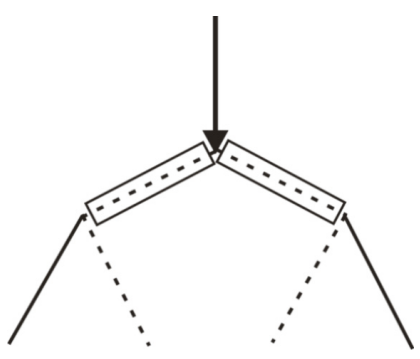

C

Fig. 3. Verification procedure for segments discarded at virtual bifurcations of PAGB: no continuation is confirmed (a) and the lost segments indicated by narrow rectangles are restored $(b, c)$. 
(Figs. 2 and 3), revealed boundaries extend again with no branching whereas the whole reconstruction is interrupted if the repetition of all the above-considered means has no effect.

\section{Results}

The local appearance of thermally etched PAGB in SEM, their panoramic image by $\mathrm{OM}$ and the PAGB reconstructed within the same area of the re-polished section are represented in Figs. $4 a-4 c$, respectively. Both the actual prior grains (Fig. 4b) and their counterparts in Fig. 4c have an almost equiaxed shape, and typical grain dimensions in these images also seem to be close.

In order to quantify the degree of similarity, respective bar charts for random intersection lengths have been composed (Fig. 5a, b) using area fractions of respective grains for partial probabilities. The most probable intersection lengths in both cases prove to be confined within the same range of 48 to $60 \mu \mathrm{m}$, whereas the related average values for the thermally etched and reconstructed PAGB are about 68 and $60 \mu \mathrm{m}$, respectively. Apparently, the above-considered comparison satisfactorily verifying the proposed algorithm could give even better results on larger areas. However, further enlargement of a single EBSD field suggests excessive time of its scanning and, hence, accumulation of much more representative data would necessitate tedious repeated measurements on numerous fields.

\section{Discussion}

Unlike conventional methods [4-10], the proposed algorithm focused on PAGB reveals dimensions and shapes of prior grains while ignoring their orientations and thus diminishing computational expenses. Austenite recrystallization can be detected in this way that is significant in consideration of hot rolling regimes. Moreover, imaging of prior grains should essentially then simplify the reconstruction of their orientations.

The selection of treated and, consequently, neglected couples of OR variants in our algorithm wants more rigorous grounds because the effect of any dropped or added couple still remains intuitive. More experiments and numerical simulations are necessary to choose the best set for analyzed combinations of OR variants. Besides, the reconstruction results notably depend on the employed OR $[5,10]$ and its fitting to EBSD data according to [11]

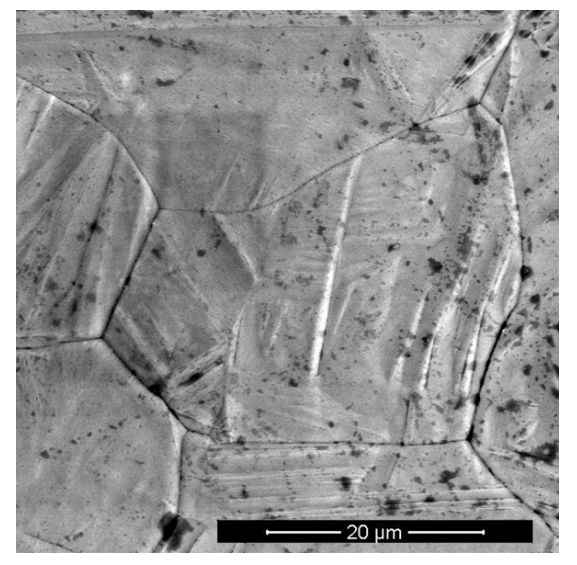

a

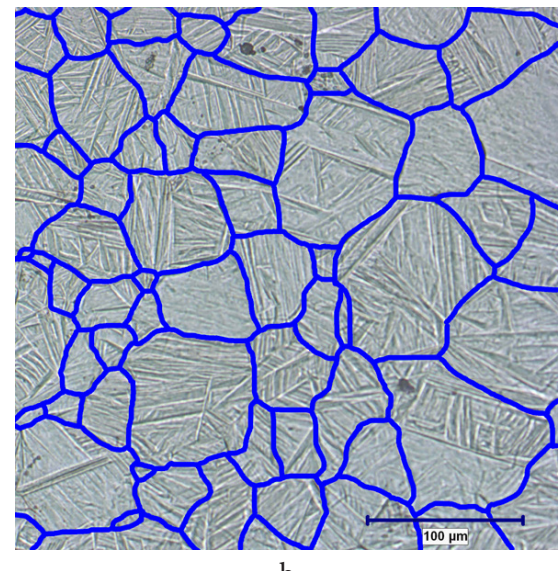

b

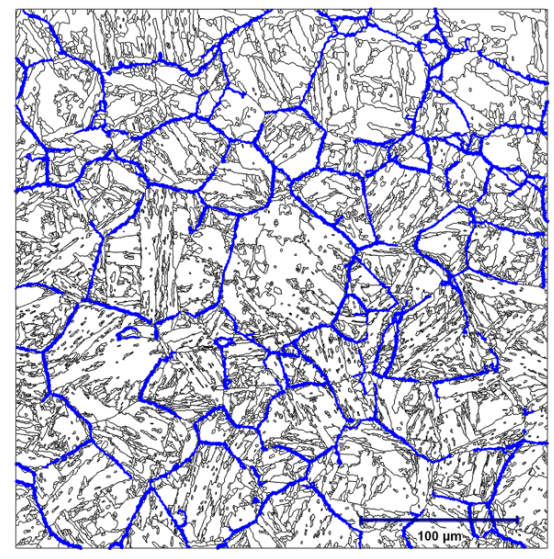

c

Fig. 4. Appearance of PAGB: SEM image of thermally etched boundaries (a), their OM image (thick lines) (b), and their counterparts reconstructed from EBSD data (c).

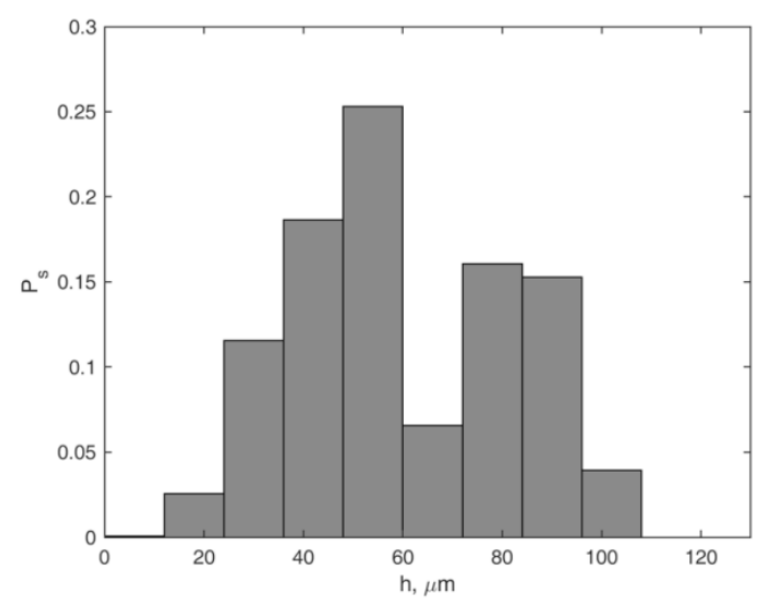

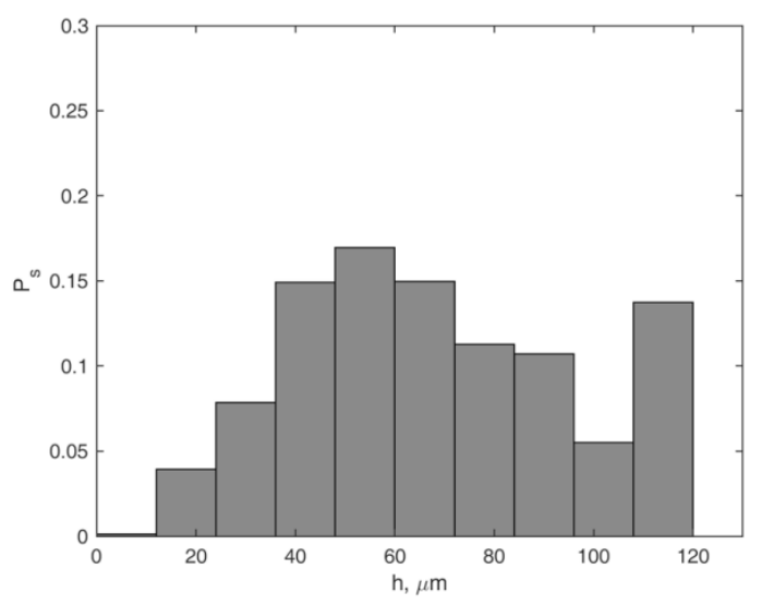

b

Fig. 5. Bar charts for lengths of random linear intersections of austenite grains in the compared structures: PAGB restored from EBSD data (a), and thermally etched prior boundaries (b). Partial probability of each bar corresponds to the area fraction of involved grains. 
also deserves consideration. Indeed, as mentioned in [5], any local OR varies even at the scale of single prior grains and, hence, a choice of determination area will influence averaged OR conventionally used to reveal PAGB. Apart from crystallographic issues, morphologic criterions could be used to correct apparent errors of the reconstruction (Fig. 4c) such as open-ended PAGB and missed third segments of virtual triple junctions.

Regardless of further improvements, the proposed method should be also verified on steels of different chemical compositions, as well as on various degrees of deformation or/and recrystallization of austenite before its transformation.

\section{Conclusion}

The novel algorithm based on particular statistics of OR variant pairing enables quick extraction of PAGB from a network of martensite interfaces revealed by EBSD. The trial results satisfactorily comply with actual prior boundaries of low carbon martensitic steel imaged by the thermal etching in vacuum. Moreover, treatment of linear rather than 2D units of the considered structure ensures much higher computational efficiency and hence a sound potential of this algorithm.

Acknowledgements. The authors thank the Ministry of education and science of the Russian Federation for support of this work within the framework of Grant 14.595.21.0004 (unique identifier RFMEF159517X0004).

\section{References}

1. C. Garcia de Andres, M. J. Bartolome, C. Capdevila, et al. Mater. Charact. 46, 389 (2001).

2. C. Garcia de Andres, F. G. Caballero, C. Capdevila, D. San
Martin. Mater. Charact. 49, 121 (2002).

3. T. V. Soshina, A. A. Zisman, E. I. Khlusova. Metallurgist 57, 128 (2013).

4. C. Cayron, B. Artaud, L. Briottet. Mater. Charact. 57, 386 (2006). DOI: 10.1016/j.matchar.2006.03.008

5. L. Germain, N. Gey, R. Mercier, P. Blaineau, M. Humbert. Acta Mater. 60, 4551 (2012).

6. Majid Abbasi, T. W. Nelson, C. D. Sorensen, L. Wei. Mater. Charact.66,1 (2012). DOI: 10.1016/j.matchar.2012.01.010

7. Majid Abbasi, Dong-Ik Kim, T.W. Nelson, Mehrdad Abbasi. Mater. Charact. 95, 219 (2014).

8. N. Bernier, L. Bracke, L. Malet, S. Godet. Mater. Charact. 89, 23 (2014).

9. A.H. Pham, T. Ohba, S. Morito, T. Hayashi. Mater. Trans. 56, 1639 (2015). DOI: 10.2320/matertrans.MAW201509

10. L. Sanz, B. Pereda, B. Lopez. Metall. Mater. Trans. 48A, 5258 (2017). DOI: 10.1007/s11661-017-4289-1

11. N.Y. Zolotorevsky, S.N. Panpurin, A.A. Zisman, S.N. Petrov. Mater. Charact. 107, 278 (2015). DOI: 10.1016/j.matchar.2015.07.023

12. S. N. Petrov, A. V. Ptashnik, Met. Sci. Heat Treatm. (2018), in print.

13. F. Archie, S. Zaefferer, Mater. Sci. Eng. A731, 539 (2018). DOI: 10.1016/j.msea.2018.06.090

14. G. Miyamoto, N. Iwata, N. Takayama, T. Furuhara. Acta Mater. 60, 1139 (2012). DOI: $10.1016 /$ j.actamat.2010.08.001

15. N. Takayama, G. Miyamoto, T. Furuhara. Acta Mater. 60, 2387 (2012).

16. A.Stormvinter, G. Miyamoto, T.Furuhara, etal. Acta. Mater. 60, 7265 (2012). DOI: 10.1016/j.actamat.2011.12.018

17. G. Kurdjumov, G. Sachs. Z. Phys. 64, 3235 (1930).

18. Z. Nishiyama. Sci. Reprts. Thohoku Univ. 23, 637 (1934).

19. A.B. Greninger, A.R. Troiano. Trans. AIME 185, 590 (1949).

20. S. Zhang, S. Morito, Y. Komizo. ISIJ Int. 52, 510 (2012). 